\title{
ON THE EXISTENCE OF SOLUTIONS WITH PRESCRIBED ASYMPTOTIC BEHAVIOUR FOR PERTURBED NONLINEAR DIFFERENTIAL EQUATIONS OF SECOND ORDER
}

\author{
OCTAVIAN G. MUSTAFA \\ Department of Mathematics, University of Craiova, Al. I. Cuza 13, Craiova, Romania \\ Department of Mathematics, University of Lund, P.O. Box 118, SE-22100 Lund, Sweden \\ e-mail:octawian@yahoo.com
}

(Received 11 May, 2004; accepted 8 September, 2004)

\begin{abstract}
A global existence result for solutions $u(t)$ of the differential equation $x^{\prime \prime}+f(t, x)=p(t), t \geq t_{0} \geq 1$, that can be written as $u(t)=P(t)+o(1)$ for all large $t$, where $P^{\prime \prime}(t)=p(t)$, is established by means of the Schauder-Tikhonov theorem. It generalizes the recent work of Lipovan [On the asymptotic behaviour of the solutions to a class of second order nonlinear differential equations, Glasgow Math. J. 45 (2003), 179-187] and allows for a unifying treatment of the existence problems concerning asymptotically linear and oscillatory solutions of second order nonlinear differential equations.
\end{abstract}

2000 Mathematics Subject Classification. 34A34, 34C10, 34E05, 47H10.

1. Introduction. In this note, we consider the perturbed nonlinear differential equation of second order

$$
x^{\prime \prime}+f(t, x)=p(t), t \geq t_{0} \geq 1
$$

where the functions $f:\left[t_{0},+\infty\right) \times \mathbb{R} \rightarrow \mathbb{R}$ and $p:\left[t_{0},+\infty\right) \rightarrow \mathbb{R}$ are continuous.

Recently, Lipovan [12] demonstrated the existence of a global solution $u(t)$ of Equation (1) that is asymptotic to a given straight line $L(t)=a t+b$, where $a, b \in \mathbb{R}$, i.e.

$$
\lim _{t \rightarrow+\infty}[u(t)-L(t)]=0
$$

Similar and related results have been obtained in [20], [14], [2], [23], [3], [19], [13], [15], [21], [22]. We mention also the pioneering contribution [1]. An investigation of the existence of such solutions, usually referred to as asymptotically linear, is essential for the oscillation theory of ordinary differential equations (see the references in [15]) as well as for the existence theory for positive solutions of semilinear elliptic problems in exterior domains (see [4], [22]).

Another important topic in the qualitative theory of ordinary and functional differential equations regarding Equation (1) is that of deriving sufficient conditions for the nonlinearity $f(t, x)$ to ensure that the oscillatory character of the perturbation $p(t)$ is inherited by all or at least by some of the solutions (say, for instance, the bounded solutions) of Equation (1). See [11], [18], [7], [10] and [16]. 
Here, by using the Schauder-Tikhonov theorem [15], we establish in rather general circumstances the existence of a global solution $u(t)$ of Equation (1) that admits the following representation

$$
u(t)=P(t)+o(1) \quad \text { as } t \rightarrow+\infty,
$$

where $P^{\prime \prime}(t)=p(t)$ for $t \geq t_{0}$. If $P(t)=a t+b$, with $a, b \in \mathbb{R}$, an extension of the results in [12] is obtained. Also, if $\liminf _{t \rightarrow+\infty} P(t)<0, \lim _{\sup _{t \rightarrow+\infty}} P(t)>0$, the existence of an oscillatory solution $u(t)$ of Equation (1) can be derived.

\section{The results.}

THEOREM 1. Assume that the nonlinearity $f(t, x)$ in Equation (1) satisfies the inequality

$$
|f(t, x)| \leq F(t,|x|), t \geq t_{0}, x \in \mathbb{R},
$$

where $F:\left[t_{0},+\infty\right) \times \mathbb{R} \rightarrow[0,+\infty)$ is a continuous function that is nondecreasing in the last argument. Suppose further that there exists a number $\varepsilon>0$ such that

$$
\int_{t_{0}}^{+\infty} s F(s,|P(s)|+\varepsilon) d s \leq \varepsilon .
$$

Then Equation (1) has a solution $u(t)$ defined in $\left[t_{0},+\infty\right)$ with the asymptotic representation (2).

Proof. We introduce the set $Y$ of all functions $y(t)$ from $C\left(\left[t_{0},+\infty\right), \mathbb{R}\right)$ such that $\lim _{t \rightarrow+\infty} t y(t)=0$. If endowed with the usual function operations and the Chebyshevtype norm

$$
\|y\|=\sup _{t \geq t_{0}}\{t|y(t)|\}
$$

$Y$ becomes a Banach space. (See [5], [21].) Let $B(\varepsilon)$ be the closed ball of radius $\varepsilon$ and center 0 in $Y$ and consider the operator $T: B(\varepsilon) \rightarrow Y$ given by

$$
[T(y)](t)=\frac{1}{t} \int_{t}^{+\infty} s f\left(s, P(s)-s \int_{s}^{+\infty} \frac{y(v)}{v} d v\right) d s, t \geq t_{0},
$$

for all $y \in B(\varepsilon)$.

By a direct computation

$$
\begin{aligned}
t|[T(y)](t)| & \leq \int_{t}^{+\infty} s F\left(s,|P(s)|+s \int_{s}^{+\infty} \frac{|y(v)|}{v} d v\right) d s \\
& \leq \int_{t}^{+\infty} s F\left(s,|P(s)|+\|y\|\left(s \int_{s}^{+\infty} \frac{d v}{v^{2}}\right)\right) d s \\
& \leq \int_{t}^{+\infty} s F(s,|P(s)|+\varepsilon) d s \leq \varepsilon
\end{aligned}
$$

we conclude that the operator $T$ is well-defined, since

$$
T(B(\varepsilon)) \subseteq B(\varepsilon) .
$$


The technique from [15] can be adapted easily to establish that the operator $T$ is completely continuous (compact). Thus, according to the Schauder-Tikhonov theorem, there exists a fixed point $y_{0}(t)$ of $T$ in $B(\varepsilon)$.

The $C^{2}$-function $u(t), t \geq t_{0}$, given by the formula

$$
u(t)=P(t)-t \int_{t}^{+\infty} \frac{y_{0}(s)}{s} d s, t \geq t_{0},
$$

is the solution of Equation (1) for which we are looking.

By application of L'Hospital's rule, we obtain

$$
\lim _{t \rightarrow+\infty} t \int_{t}^{+\infty} \frac{y_{0}(s)}{s} d s=\lim _{t \rightarrow+\infty} t y_{0}(t)=0 .
$$

The proof is complete.

COROLlary 2. Consider the nonlinear differential equation

$$
x^{\prime \prime}+f(t, x)=0, \quad t \geq t_{0} \geq 1,
$$

and assume that the following inequality is valid:

$$
\int_{t_{0}}^{+\infty} s F(s,|a s+b|+\varepsilon) d s \leq \varepsilon
$$

for certain $a, b \in \mathbb{R}$, where $F(t, z)$ is given by (3). Then, Equation (6) has a solution $u(t)$ defined in $\left[t_{0},+\infty\right)$ that is asymptotic to the straight line $L(t)=a t+b$; that is

$$
\lim _{t \rightarrow+\infty}[u(t)-L(t)]=0 .
$$

Proof. We take $P(t)=a t+b$ and apply Theorem 1 .

COROLlary 3. Suppose that (4) holds and, simultaneously, there exists an increasing sequence $\left(t_{n}\right)_{n \geq 1}$, with $t_{1} \geq t_{0}$, such that $\left(t_{n}\right)_{n \geq 1}$ is not bounded above and

$$
P\left(t_{2 n-1}\right)>\varepsilon \quad P\left(t_{2 n}\right)<-\varepsilon, \quad n \geq 1 .
$$

Then Equation (1) has an oscillatory solution $u(t)$ defined in $\left[t_{0},+\infty\right)$.

Proof. From (5) we deduce that

$$
|u(t)-P(t)| \leq \varepsilon, t \geq t_{0} .
$$

Then

$$
u\left(t_{2 n-1}\right) \geq P\left(t_{2 n-1}\right)-\varepsilon>0
$$

and

$$
u\left(t_{2 n}\right) \leq P\left(t_{2 n}\right)+\varepsilon<0,
$$

for all $n \geq 1$. The existence of a zero of $u(t)$ in $\left(t_{2 n-1}, t_{2 n}\right)$ is a consequence of the continuity of the solution. 
EXAMPLE 4. Fix $c>0, \varepsilon \in(0,3]$. Let $p \in C\left(\left[t_{0},+\infty\right), \mathbb{R}\right)$ be nonnegative. Introduce $P, t_{0}$ by the formulae

$$
P(t)=c+\int_{t_{0}}^{t}(t-s) p(s) d s, t \geq t_{0}
$$

and

$$
t_{0}=\frac{3}{\varepsilon}\left(1+\frac{\varepsilon}{c}\right)^{2} \geq 1
$$

The nonlinearity $f(t, x)$ of the Emden-Fowler equation below

$$
x^{\prime \prime}-\frac{2}{t[t P(t)+1]^{2}} x^{2}=p(t), t \geq t_{0},
$$

satisfies the hypotheses of Theorem 1. In fact, condition (4) reads as

$$
\begin{aligned}
\int_{t_{0}}^{+\infty} \frac{2}{s^{2}}\left(\frac{P(s)+\varepsilon}{P(s)+s^{-1}}\right)^{2} d s & \leq \int_{t_{0}}^{+\infty} \frac{2}{s^{2}}\left(1+\frac{\varepsilon}{c}\right)^{2} d s \\
& =\frac{2}{t_{0}}\left(1+\frac{\varepsilon}{c}\right)^{2}<\varepsilon .
\end{aligned}
$$

It is easy to see that Equation (10) has the exact solution $u(t)=P(t)+t^{-1}$ for $t \geq t_{0}$.

Let us employ now the integral operator $T$ given in Theorem 1 to give an alternative proof of a general existence result for the asymptotically linear solutions of Equation (6). See [15] and [12]. The proof relies on the fixed point theorem referred to as the Leray-Schauder alternative [6], [15].

COROLlary 5. Suppose that there exist continuous functions $h_{1}, h_{2}:\left[t_{0},+\infty\right) \rightarrow$ $[0,+\infty)$ and $g:[0,+\infty) \rightarrow(0,+\infty)$ such that

$$
F(t, z)=h_{1}(t) g\left(\frac{z}{t}\right)+h_{2}(t), t \geq t_{0}, z \geq 0 .
$$

Assume further that $g(w)$ is nondecreasing and

$$
\int_{0}^{+\infty} \frac{d w}{g(w)}=+\infty, \quad \int_{t_{0}}^{+\infty} s h_{i}(s) d s<+\infty, i=1,2 .
$$

Then for any $a, b \in \mathbb{R}$, Equation (6) has a solution $u(t)$ defined in $\left[t_{0},+\infty\right)$ such that (8) holds.

Proof. Introduce $P(t)=L(t)$ for $t \geq t_{0}$. According to the Leray-Schauder alternative, in order to establish that the integral operator $T$ defined in the proof of Theorem 1 has a fixed point we have to show that the set

$$
E(T)=\{y \in Y: y=\lambda T(y) \text { for a certain } 0<\lambda<1\}
$$

is bounded. In fact, for $y \in E(T)$, we deduce that

$$
t|y(t)| \leq H+\int_{t}^{+\infty} \operatorname{sh}_{1}(s) g\left(|a|+|b|+\int_{s}^{+\infty} \frac{|y(v)|}{v} d v\right) d s,
$$

for all $t \geq t_{0}$, where $H=\int_{t_{0}}^{+\infty} s h_{2}(s) d s$. 
Using integration by parts, we obtain

$$
\begin{aligned}
\int_{t}^{+\infty} \frac{|y(s)|}{s} d s \leq & H t_{0}^{-1}+\int_{t}^{+\infty} \frac{1}{s^{2}} \int_{s}^{+\infty} v h_{1}(v) g\left(|a|+|b|+\int_{v}^{+\infty} \frac{|y(w)|}{w} d w\right) d v d s \\
\leq & H+\frac{1}{t} \int_{t}^{+\infty} \operatorname{sh}_{1}(s) g\left(|a|+|b|+\int_{s}^{+\infty} \frac{|y(v)|}{v} d v\right) d s \\
& -\int_{t}^{+\infty} h_{1}(s) g\left(|a|+|b|+\int_{s}^{+\infty} \frac{|y(v)|}{v} d v\right) d s
\end{aligned}
$$

and so

$$
z(t) \leq K+\int_{t}^{+\infty} \operatorname{sh}_{1}(s) g(z(s)) d s, t \geq t_{0},
$$

where $z(t)=|a|+|b|+\int_{t}^{+\infty} \frac{|y(s)|}{s} d s$ and $K=H+|a|+|b|$.

According to [15], we deduce that

$$
z(t) \leq Z(t)=G^{-1}\left(G(K)+\int_{t}^{+\infty} s h_{1}(s) d s\right)<+\infty,
$$

where $G(x)=\int_{0}^{x} \frac{d w}{g(w)}$ for all $x \geq 0$.

In conclusion,

$$
\|y\| \leq H+g\left(Z\left(t_{0}\right)\right) \int_{t_{0}}^{+\infty} s h_{1}(s) d s, y \in E(T) .
$$

The proof is complete.

REMARK 1. The Leray-Schauder alternative and condition (12) 1 were needed only to ensure the global existence of the asymptotically linear solution $u(t)$. If, as in [12], the solution $u(t)$ is allowed to exist only for large $t$, then Corollary 5 follows from Theorem 1 for an appropriate choice of $t_{0}$. In fact, in this case (4) should read as

$$
g(|a|+|b|+\varepsilon) \int_{t_{0}}^{+\infty} s h_{1}(s) d s+\int_{t_{0}}^{+\infty} s h_{2}(s) d s \leq \varepsilon .
$$

REMARK 2. Corollary 2 complements [22]. In fact, if for a certain $c>0$ we have

$$
\int_{t_{0}}^{+\infty} t F(t, 2 c t) d t<+\infty
$$

then, for a $t_{1} \geq t_{0}$ sufficiently large, condition (7) reads as $(a=c, b=0, \varepsilon=c)$

$$
\int_{t_{1}}^{+\infty} s F(s, c s+c) d s \leq \int_{t_{1}}^{+\infty} s F(s, 2 c s) d s<c
$$

and Equation (6) has a solution $u(t)$ defined in $\left[t_{1},+\infty\right)$ such that

$$
u(t)=c t+o(1) \quad \text { as } t \rightarrow+\infty .
$$

REMARK 3. It is not clear from Corollary 3 whether the oscillatory solution $u(t)$ tends to zero as $t \rightarrow+\infty$ or the quantity $\lim _{t \rightarrow+\infty} u(t)$ does not exist. However, by 
replacing in Theorem 1 hypothesis (4) with the following inequality

$$
\int_{t}^{+\infty} s F(s,|P(s)|+q(s)) d s \leq q(t), \quad t \geq t_{0},
$$

where $q \in C\left(\left[t_{0},+\infty\right), \mathbb{R}\right)$ decreases to zero as $t \rightarrow+\infty$, the set $B(\varepsilon)$ with the one below

$$
B_{q}=\left\{y \in Y: t|y(t)| \leq q(t) \text { for all } t \geq t_{0}\right\}
$$

and hypothesis (9) with

$$
\left\{\begin{array}{cl}
P\left(t_{2 n-1}\right)>q\left(t_{2 n-1}\right) \quad & P\left(t_{2 n}\right)<-q\left(t_{2 n}\right), \quad n \geq 1, \\
\lim _{t \rightarrow+\infty} P(t)=0 &
\end{array}\right.
$$

the existence of an oscillatory solution $u(t)$ of Equation (1) such that $\lim _{t \rightarrow+\infty} u(t)=0$ follows from Corollary 3.

REMARK 4. Obtaining asymptotic integration results via fixed point theory usually leads to special, sometimes complicated, function spaces; see [15], [21], [22]. The function space employed in [12] is simple. However, the proof relies on a change of variables similar to the one suggested in [9]. The function space $Y$, introduced here, is closer to the ideas developed in [8]. This allows us to establish Theorem 1 in a direct way. As a by-product, in the case of $P(t)=a t$, where $a \in \mathbb{R}$, (see [23], [13], [22]) the function $y_{0}(t)$ reads as $u^{\prime}(t)-t^{-1} u(t)$, a quantity playing a significant role in asymptotic integration theory [17].

A careful inspection of proofs from [14], [2], [19], [15] shows that, if (12), holds, all solutions of Equation (6) are defined globally in the future and satisfy (8) for appropriate $a, b \in \mathbb{R}$. As opposed to this situation, the violation of condition (12) 1 leads to solutions that either blow up in finite time or are not asymptotic to straight lines. See [15] and [17].

ExAmple 6. Consider the differential equation below

$$
u^{\prime \prime}=(3-t) e^{-t} u^{2}+(4-t) e^{-2 t} u^{3}, \quad t \geq t_{0}=1 .
$$

Here, $h_{1}(t)=t^{3} e^{-t}(|3-t|+|4-t|), h_{2}(t)=0$ and $g(z)=1+z^{2}+z^{3}$. Obviously, (12) 1 is not valid. Equation (13) has the exact solution

$$
u(t)=\frac{e^{t}}{2-t}, \quad t \in[1,2),
$$

that cannot be continued to the right of $t=2$.

EXAMPLE 7. The differential equation

$$
u^{\prime \prime}=e^{-t} u^{2}, \quad t \geq t_{0}=1,
$$

has the exact solution

$$
u(t)=e^{t}, \quad t \geq 1,
$$

which is not asymptotically linear. Here, $h_{1}(t)=t^{2} e^{-t}, h_{2}(t)=0$ and $g(z)=1+z^{2}$. The condition $(12)_{1}$ is violated. 
The next result gives a hint of the asymptotic behaviour of solutions of Equation (6) when condition $(12)_{2}$ is replaced with a weaker one.

THEOREM 8. Assume that the nonlinearity $f(t, x)$ in Equation (6) satisfies the inequality (3). Suppose further that there exist numbers $a \in \mathbb{R}, c \in(0,1)$ and $\varepsilon>0$ such that

$$
\int_{t_{0}}^{+\infty} s^{c} F\left(s,\left(|a|+\frac{\varepsilon}{c} t_{0}^{-c}\right) s\right) d s \leq \varepsilon .
$$

Then Equation (6) has a solution $u(t)$ defined in $\left[t_{0},+\infty\right)$ with the asymptotic representation

$$
u(t)=a t+o\left(t^{1-c}\right) \quad \text { as } t \rightarrow+\infty .
$$

Proof. We introduce the set $Z$ of all functions $z(t)$ from $C\left(\left[t_{0},+\infty\right), \mathbb{R}\right)$ such that $\lim _{t \rightarrow+\infty} t^{c} z(t)=0$. If endowed with the usual linear operations and the Chebyshevtype norm

$$
\|z\|=\sup _{t \geq t_{0}}\left\{t^{c}|z(t)|\right\}
$$

$Z$ becomes a Banach space. Let $B(\varepsilon)$ be the closed ball of radius $\varepsilon$ and center 0 in $Z$ and consider the operator $T: B(\varepsilon) \rightarrow Z$ given by

$$
[T(z)](t)=-\frac{1}{t} \int_{t_{0}}^{t} s f\left(s, a s-s \int_{s}^{+\infty} \frac{z(v)}{v} d v\right) d s, t \geq t_{0},
$$

for all $z \in B(\varepsilon)$.

Hypothesis (14) yields

$$
\int_{t_{0}}^{t} s F\left(s,|a| s+\frac{\varepsilon}{c} s^{1-c}\right) d s \leq \varepsilon t^{1-c}, t \geq t_{0} .
$$

This follows from

$$
\begin{aligned}
\varepsilon & \geq \int_{t_{0}}^{t} s^{c} F\left(s,\left(|a|+\frac{\varepsilon}{c} t_{0}^{-c}\right) s\right) d s \geq \int_{t_{0}}^{t} s^{c} F\left(s,|a| s+\frac{\varepsilon}{c} s^{1-c}\right) d s \\
& \geq \int_{t_{0}}^{t} \frac{s}{t^{1-c}} F\left(s,|a| s+\frac{\varepsilon}{c} s^{1-c}\right) d s .
\end{aligned}
$$

By direct computation

$$
\begin{aligned}
t^{c}|[T(z)](t)| & \leq \frac{1}{t^{1-c}} \int_{t_{0}}^{t} s F\left(s,|a| s+s \int_{s}^{+\infty} \frac{|z(v)|}{v} d v\right) d s \\
& \leq \frac{1}{t^{1-c}} \int_{t_{0}}^{t} s F\left(s,|a| s+\|z\|\left(s \int_{s}^{+\infty} \frac{d v}{v^{1+c}}\right)\right) d s \\
& \leq \frac{1}{t^{1-c}} \int_{t_{0}}^{t} s F\left(s,|a| s+\frac{\varepsilon}{c} s^{1-c}\right) d s \leq \varepsilon,
\end{aligned}
$$

we deduce that the operator $T$ is well-defined since $T(B(\varepsilon)) \subseteq B(\varepsilon)$.

The proof can now be completed in the same way as the proof of Theorem 1 . 
Corollary 9. Suppose that (3) and (11) hold. Assume further that

$$
\int_{t_{0}}^{+\infty} s^{c} h_{i}(s) d s<+\infty, i=1,2,
$$

for a certain $c \in(0,1)$. Then, for any $a \in \mathbb{R}$ there exist a number $t_{a} \geq t_{0}$ and a solution $u(t)$ of Equation (6) defined in $\left[t_{a},+\infty\right)$ satisfying (15).

Proof. Introduce $H_{i}(t)=t^{c} h_{i}(t)$ for $t \geq t_{0}$. Then, (16) reads as

$$
\begin{aligned}
& g\left(|a|+\frac{\varepsilon}{c}\right) \frac{1}{t^{d}} \int_{t_{0}}^{t} s^{d} H_{1}(s) d s+\frac{1}{t^{d}} \int_{t_{0}}^{t} s^{d} H_{2}(s) d s \\
\leq & g\left(|a|+\frac{\varepsilon}{c}\right) \int_{t_{0}}^{+\infty} H_{1}(s) d s+\int_{t_{0}}^{+\infty} H_{2}(s) d s \\
\leq & \varepsilon,
\end{aligned}
$$

where $d=1-c$.

ACKnOwledgements. The author is deeply indebted to Professor Adrian Constantin of the University of Lund for calling his attention to reference [3] and to the referee for careful and thoughtful reading of the manuscript and useful suggestions which helped to improve the presentation of results.

\section{REFERENCES}

1. I. Bihari, Researches of the boundedness and stability of the solutions of non-linear differential equations, Acta Math. Acad. Sci. Hung. 8 (1957), 261-278.

2. A. Constantin, On the asymptotic behaviour of second order nonlinear differential equations, Rend. Mat. Appl. 13 (1993), 627-634.

3. A. Constantin, Positive solutions of Schrödinger equations in two-dimensional exterior domains, Monatsh. Math. 123 (1997), 121-126.

4. A. Constantin and G. Villari, Positive solutions of quasilinear elliptic equations in two-dimensional exterior domains, Nonlin. Anal. 42 (2000), 243-250.

5. C. Corduneanu, Integral equations and stability of feedback systems (Academic Press, New York, 1973).

6. J. Dugundji and A. Granas, Fixed point theory, I, Monogr. Matem., Tome 61 (PWN, Warszawa, 1982).

7. J. R. Graef, M. K. Grammatikopoulos and P. W. Spikes, On the decay of oscillatory solutions of a forced higher order functional differential equation, Math. Nachr. 117 (1984), $141-153$.

8. J. K. Hale and N. Onuchic, On the asymptotic behaviour of solutions of a class of differential equations, Contrib. Diff. Eqs. 2 (1963), 61-75.

9. P. Hartman and N. Onuchic, On the asymptotic integration of ordinary differential equations, Pacific J. Math. 13 (1963), 1193-1207.

10. G. Karakostas, I. P. Stavroulakis and Y. Wu, Oscillations of Volterra integral equations with delay, Tohoku Math. J. 45 (1993), 583-605.

11. A. G. Kartsatos, On the maintenance of oscillation of $n$-th order equations under the effect of a small forcing term, J. Diff. Eqs. 10 (1971), 355-363.

12. O. Lipovan, On the asymptotic behaviour of the solutions to a class of second order nonlinear differential equations, Glasgow Math. J. 45 (2003), 179-187.

13. S. Masmoudi and N. Yazidi, On the existence of positive solutions of a singular nonlinear differential equation, J. Math. Anal. Appl. 268 (2002), 53-66. 
14. F. W. Meng, A note on Tong's paper: The asymptotic behaviour of a class of nonlinear differential equations of second order, Proc. Amer. Math. Soc. 108 (1990), 383-386.

15. O. G. Mustafa and Yu. V. Rogovchenko, Global existence of solutions with prescribed asymptotic behaviour for second-order nonlinear differential equations, Nonlin. Anal. 51 (2002), 339-368.

16. O. G. Mustafa and Yu. V. Rogovchenko, Oscillation of second-order perturbed nonlinear differential equations, Math. Nachr., to appear.

17. O. G. Mustafa and Yu. V. Rogovchenko, Global existence and asymptotic behaviour of solutions of nonlinear differential equations, Funkc. Ekvac. 47 (2004), 167-186.

18. C. G. Philos, Oscillatory and asymptotic behaviour of the bounded solutions of differential equations with deviating arguments, Hiroshima Math. J. 8 (1978), 31-48.

19. S. P. Rogovchenko and Yu. V. Rogovchenko, Asymptotic behaviour of solutions of second order nonlinear differential equations, Portugal. Math. 57 (2000), 17-33.

20. J. Tong, The asymptotic behaviour of a class of nonlinear differential equations of second order, Proc. Amer. Math. Soc. 84 (1982), 235-236.

21. B. Yan, Multiple unbounded solutions of boundary value problems for second-order differential equations on the half-line, Nonlin. Anal. 51 (2002), 1031-1044.

22. Z. Yin, Monotone positive solutions of second-order nonlinear differential equations, Nonlin. Anal. 54 (2003), 391-403.

23. Z. Zhao, Positive solutions of nonlinear second order ordinary differential equations, Proc. Amer. Math. Soc. 121 (1994), 465-469. 